\title{
Phase-Locked Particle Image Velocimetry Visualization of the Sound Field at the Outlet of a Circular Tube
}

\author{
S. WEYNA AND W. MiCKIEWICZ* \\ West Pomeranian University of Technology, al. Piastów 17, 70-310 Szczecin, Poland
}

\begin{abstract}
The shedding process and acoustic flow formation in the region of outlet of a cylinder duct has been investigated by means of the particle image velocimetry (PIV) technique. The research work on the behavior of the sound field at outlets of waveguides addresses issues of importance to industrial applications. The obtained results can be used in a number of industrial applications involving pipes, ducts and tubing. In our investigation the acoustics flow fields are measured with a phase-locked PIV system with high sampling rate and large internal memory block using DAVIS v. 8.11 software. A cross-correlation method, in conjunction with the FFT analysis, is used as a vector processing algorithm. A series of PIV vector maps was used to evaluate the acoustic velocity field at the open end of circular tube throughout the acoustic cycle for frequencies of $700 \mathrm{~Hz}$ and $1400 \mathrm{~Hz}$. Six phase steps were assumed for one frequency cycle and the characteristic signal was extracted from the velocity data step and shown graphically.
\end{abstract}

DOI: $10.12693 /$ APhysPolA.125.A-108

PACS: 43.58.+z, 43.28.Js, 43.25.-x, 47.35.Rs

\section{Introduction}

The radiation of sound from open end of circular resonant tube is relatively well understood at low amplitudes and quite widely described in the literature. Understanding the mechanism of wake formation in relation to the object geometry and flow conditions is a major research topic in the field of bluff body aerodynamics [1-3]. However, in many technical applications, the problem of sound and noise generation is still an inspiration for research [4]. This has implications in a number of fields, notably in the automotive industry and in musical instrument design. For non-invasive examination of the acoustic field distribution at waveguide outlets, anemometric laser techniques are being used today [5]. One of them is an optical technique called the particle image velocimetry (PIV) used for measurement of whole-field instantaneous velocity data [6]. This technique does not disturb the flow and offers the benefit of allowing both qualitative and quantitative description of the flow field to be made. PIV technique is a tool more and more commonly used in both scientific research and engineering practice. Fields of its application include flows in the fields of aerodynamics, air-conditioning and ventilation systems, chemical pipelines installations, and most recently, also acoustics flow fields measurements. In this paper we present our results obtained in the area of the sound field PIV visualization. We have investigated the sound source in the form of outlet of a circular pipe driven by a dynamic loudspeaker.

\section{PIV investigating technique adapted to acoustics}

The objective of our researches was determined on the basis of a review of current knowledge on the acoustic the-

*corresponding author; e-mail: witold.mickiewicz@zut.edu.pl ory of vortex flow and turbulent noise generation mechanism (from Lighthill 1952 [7] to Howe 2008 [8]). In our studies we shall put particular emphasis on specific topics relevant to the new perception of acoustic flow field theory supported by experimental results achieved with the use of physical models by means of laser anemometry techniques.

Laser anemometry is a method which allows to take non-intrusive instantaneous measurements of vector fields. This non-intrusiveness of laser-based measurement methods represents the greatest advantage against other methods using sensors for flow measurements. Laser methods are extensively used nowadays in fluid dynamics to measure many quantities on the grounds of the particle motion picture. PIV was first developed for fluid mechanics measurements and their application to pure acoustic flow is rather rarely reported in scientific literature in comparison with mass flow experiments $[5,9,10]$. So far, the vector acoustics was supported only by intrusive measurements of the sound intensity vector field using pressure-pressure or pressure-velocity probes [11]. Properly adapted laser methods can also be used to provide information about instantaneous flow and acoustic velocities. Adding some numerical methods for acoustic pressure estimation makes it possible to obtain sound intensity maps with the minimum disturbance of the sound field [12].

PIV is not the only laser technique used for velocity measurements. The Laser Doppler Velocimetry (LDV) is another method in which acoustic flow velocity measurements are based on random sampling of velocity events occurring when the so-called seeding particles pass through the measurement volume. The available velocity sampling rate depends on particle concentration, particle size, flow velocity and other flow-related and optical parameters. This method will be not addressed in the present paper. 
The development of particle image velocimetry (PIV) measurement technique consisting in capturing instantaneous velocity information of whole flow fields has been developed in the eighties of 20th century. Over the years the hardware and software supporting PIV technique have developed, while image recording devices, light sources, optics and image processing significantly improved [1, 2, 13]. Nowadays, PIV is applied in still growing number of research and technology areas: from aerodynamics to biology, from fundamental turbulence research to applications in the turbo-machinery design, from combustion to two phase flows and, particularly intensively, in micro devices and systems. Due to the variety of different applications of PIV and the large number of different possibilities to illuminate, record and evaluate, many different dedicated technical modifications of the PIV technique have been developed. Moreover, numerous publications describe the problems from a specific point of view (eg. in fluid mechanics). In our research we focus on acoustic PIV (A-PIV) application to visualize acoustic velocity of the sound field at the outlet of a circular tube. The results are important from the point of view of theoretical and applied acoustics and allow to see invisible acoustic energy flow.

Figure 1 shows a schematic diagram of the Particle Image Velocimetry measurement system, which consists of several subsystems. If we want to visualize acoustic motions in air (or in other transparent medium), seeding particles have to be added to the investigated volume to quantify the velocity field of the fluid. These particles are illuminated by means of the so-called laser sheet in the acoustic flow plane at least twice within a short time interval $-\Delta t$ - between consecutive pulses emitted by a light source (usually a double pulse Nd:YAG laser). The light scattered by tracer particles is recorded either on a single frame or on a pair of frames on a special highresolution digital CCD or CMOS camera.

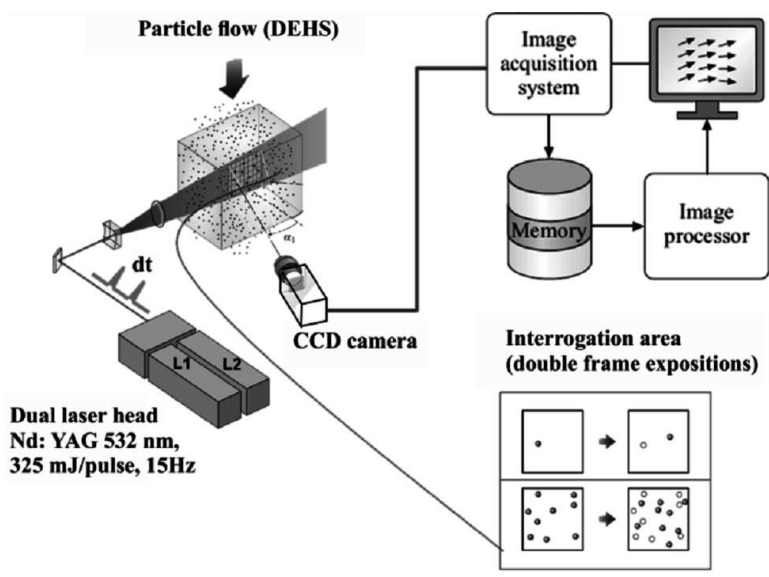

Fig. 1. Schematic diagram of planar (flat flow) Particle Image Velocimetry measurement system.

The digital PIV recording pairs are divided into small subareas called "interrogation areas". For each interroga- tion area the two-component local flow velocity vector is determined using correlation analysis and projected onto the light sheet plane. Vectors calculated for each of these interrogation areas (which can be partially overlapped) are used to reconstruct the instantaneous velocity vector field. The processing algorithms have usually some steps and are defined using the processing pipeline with a complete range of image processing tools. Innovative algorithm design and a comprehensive range of image pre-processing, processing, analysis, and display options are critical for accurate velocity measurements in the full range of potential applications. Detailed description of classical PIV systems and their application to mass flow research in various areas are presented, for example, in the book [14].

The application of PIV method to acoustics is not straightforward. In general, sound measurements have both shortcomings and advantages in comparison with classical fluid mechanics measurements. The shortcomings come from the fact that acoustic velocities and displacements are generally very small (for instance, at $1000 \mathrm{~Hz}$ and the acoustic level of $100 \mathrm{~dB}$ SPL, the velocity amplitude is about $5 \mathrm{~mm} / \mathrm{s}$ and the displacement amplitude is about $10^{-6} \mathrm{~m}$ ) and vary rapidly in time. The advantage seems to be the repetitiveness of all dynamic processes within the cycle of excitation signal. The basic objective of our research is the refinement of measurement and evaluation techniques and tools that can be used for the visualization of complex acoustic flows. PIV methods thus could find their wide application in measurements of internal acoustic flows such as those occurring in ducts and turbo-machines.

\section{Research with phase-locked PIV system}

It is difficult to observe acoustic phenomena using image techniques due to their high variability in time related to high frequencies involved. Widely used PIV systems with low image acquisition rate (about $15 \mathrm{~Hz}$ ) can not be used directly in asynchronous mode. The problem can be solved by proper synchronization of the image acquisition process with the harmonic excitation signal. Measurement of an instantaneous acoustic velocity in each phase of acoustic tone signal with time resolution meeting requirements of sampling theorem can be realized by multiple signal acquisition in time instants synchronized with excitation signal and properly delayed [5]. The measured particle displacement $-d-$ is different for each phase step, therefore the evaluated instantaneous particles velocity $-u-$ also varies sinusoidally. Image pairs acquired at consecutive phases are collected with properly increased delay, as shown in Fig. 2.

The time parameter $T_{S S}$ is the period of acquisition triggering. The trigger signal is usually synchronized with acoustic excitation. The minimum acceptable $T_{S S}$ depends on PIV system elements performance: maximum image acquisition rate of CCD camera and maximum rate of laser impulses. Acquisition of the first image in the pair is made after time $T_{D}$ from the trigger. At this moment Laser 1 generates light pulse. After chosen 


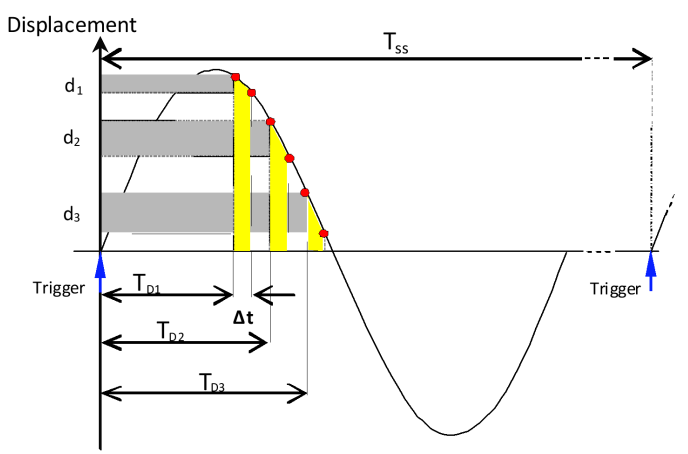

Fig. 2. Phase-locked PIV acquisition. Measured displacement variation due to the selected delay time $T_{D}$ (phase).

time interval $\Delta t$, the second pulse of Laser 2 is generated and then the second image in the pair is acquired. Now the image data can be transferred to the frame grabber and lasers can be charged. After time $T_{S S}$, the image acquisition process repeats. To achieve the required signal to noise ratio, for single acoustic signal phase (one $T_{D}$ value) many image pairs are collected (in our research 100 image pairs per phase). Next, $T_{D}$ value is being increased and the whole procedure repeated. The number of phases measured for one acoustic signal period depends on the observed phenomena and the required accuracy. At the monotonic acoustic excitation the number of phases ranges from a few to twenty. For each phase step, the particle displacement $-d-$ and the instantaneous particles velocity - $u-$ change.

\section{Experimental setup}

The work described in this paper concerns the measurement of acoustic velocity fields at the open end of a tube, in which high amplitude sound field is generated by a loudspeaker. The acoustic chamber in which the radiating tube is placed, is a Plexiglas channel of square cross-section $150 \mathrm{~mm} \times 150 \mathrm{~mm}$. The walls of the channel are $8 \mathrm{~mm}$ thick. The optical PIV technique was used to acquire two-dimensional full-field instantaneous velocity information of the acoustic velocity field at the open end in a mid-plane of $127 \mathrm{~mm}$ long tube with $13 \mathrm{~mm}$ inner diameter in which a high amplitude sound field (130 dB SPL) was generated by a loudspeaker. DEHS (Di-Ethyl-Hexyl-Sebacat, $\rho_{D E H S}=912 \mathrm{~kg} \mathrm{~m}^{-3}$ ) oil mist droplets with average diameter of $1 \mu \mathrm{m}$ were used as tracer particles. An aerosol generator (atomizer by LaVision GmbH, Goettingen, Germany) was used to generate the mist. The flow was illuminated in the observation plane by a Nd:YAG, $325 \mathrm{~mJ} /$ pulse double pulsed Litron laser model Nano TRL 325-15 with green color light source $(\lambda=532 \mathrm{~nm})$. A digital 4 megapixel progressive scan $(14 \mathrm{f} / \mathrm{s})$ CCD camera (Imager Pro X $4 \mathrm{M}$, LaVision) was used to acquire raw PIV image for the study. This camera can take separate images at resolution of 2048 x 2048 pixels with the minimum interval of $200 \mathrm{~ns}$ between images. It was possible to program the delay, relative to an input trigger signal, and the exposure time of the first image. The second exposure is continued for the time depending on how long it takes for the computer to process the first image. In our PIV system the real sampling rate of image pair acquisition is about $7 \mathrm{~Hz}$.

The camera was connected to a PC equipped with frame grabber (Matrox Solios eCL CameraLink) that acquired 14 bit images at the rate of $14 \mathrm{~Hz}(7 \mathrm{~Hz}$ rate for image pairs). A Programmable Timing Unit (PTU 9, LaVision) was used to control the timing of the laser pulses. The image pairs acquisition was phase-locked with respect to the acoustic cycle by using a Programmable Timing Unit. The CCD camera was positioned perpendicularly to the light sheet and focused on the illuminated particle mist. The field of view of the CCD camera was set in a way allowing to map the flow field around of the tube termination (120 mm horizontally by $120 \mathrm{~mm}$ vertically). A special loudspeaker driver (STX-D1500) was used to excite the acoustic wave inside the tube. The driver had a diaphragm of diameter $51 \mathrm{~mm}$, maximum power of $200 \mathrm{~W}$, and impedance of $8 \mathrm{Ohm}$. The use of a loudspeaker driver as an acoustic source (exciting $130 \mathrm{~dB}$ SPL) made it easy to vary the frequency of excitation continuously and precisely. A function generator model Agilent 33210A was used to generate the sinusoidal wave of different frequencies and amplitudes. The accuracy of the generated frequency and amplitude were $1 \mu \mathrm{Hz}$ and $1 \mathrm{mVpp}$, respectively. The signal from the function generator was amplified by a $2 \times 375 \mathrm{~W}$ amplifier (CROWN XLS2000) and supplied to the loudspeaker. In our research the frequency was set equal to $700 \mathrm{~Hz}$ (the first mode of one-end open pipe $127 \mathrm{~mm}$ long and $13 \mathrm{~mm}$ in diameter) and $1400 \mathrm{~Hz}$ (the second harmonic of the first mode). All experiments were conducted in air at $21^{\circ} \mathrm{C}$. The properties of air in the course of the measurement were as: speed of sound $c_{0}=344 \mathrm{~m} \mathrm{~s}^{-1}$, density $\rho_{0}=1.29 \mathrm{~kg} \mathrm{~m}^{-3}$ and kinematic viscosity coefficient $\nu=1.6 \times 10^{-5} \mathrm{~m}^{2} \mathrm{~s}^{-1}$.

In our experiment, the acoustic cycle was divided into 48 phases and for each phase, 75 image pairs were collected, transformed to vector field, and averaged to determine one instantaneous velocity vector field. The time interval between two consecutive images in one pair was set to $1 / 8$ of the excitation signal cycle: $179 \mu$ s for $700 \mathrm{~Hz}$ tone and $89 \mu \mathrm{s}$ for $1400 \mathrm{~Hz}$ tone. The choice of time interval value was based on [15].

Example of two instantaneous velocity vector fields for two different phases of acoustic cycle for the frequency of $1400 \mathrm{~Hz}$ is shown in Fig. 3.

As mentioned earlier, DEHS droplets were used as tracer particles for PIV measurements. The image of the tube outlet surrounded by seeding particles taken by the PIV camera is shown in Fig. 4. Acoustic velocity fields presented in the following figures correspond to the area depicted in Fig. 4.

It is necessary to be certain that the droplets faithfully follow the motion of the flow. An important issue related 


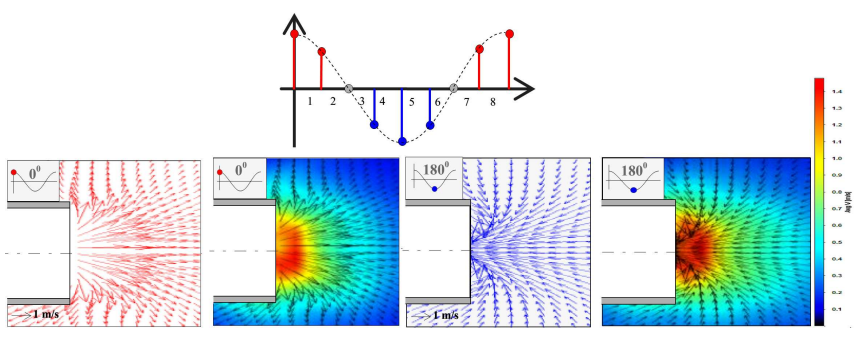

Fig. 3. Example of phase-locked instantaneous velocity vector field in two different phases of a single acoustic cycle for the $1400 \mathrm{~Hz}$ tone.

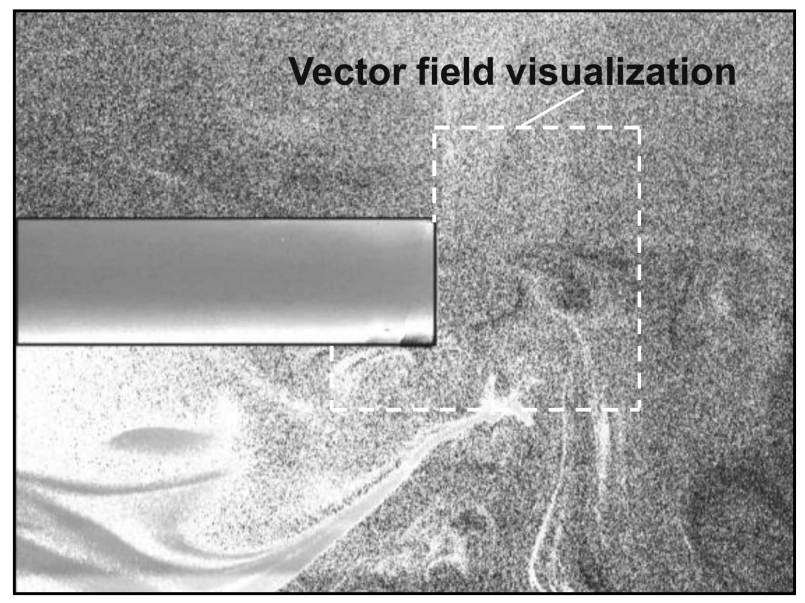

Fig. 4. Image of seeding particles around the outlet of the investigated tube.

to the tracer particles motion abilities is their response time determining how quickly the particles respond to any change in the flow behavior. In the present study, the density of the tracer particles was much higher than that of the air; therefore, it is important to find the response time of the actually used tracer particles.

The characteristic response time of seeding particles is computed by

$$
T_{\mathrm{p}}=\frac{u_{\mathrm{T}}}{g},
$$

where $T_{\mathrm{p}}$ is the particle response time, $u_{\mathrm{T}}$ is the particle terminal velocity and $g$ is the gravity acceleration [16].

The terminal velocity can be calculated as:

$$
u_{\mathrm{T}}=\frac{(\gamma-1) D^{2} g}{18 \nu},
$$

where $D$ is the diameter of a tracer particle, $\nu$ is the kinematic viscosity of the fluid, and $\gamma$ is the ratio of the density of particle to the density of fluid [17]. Using the above equations, for $D=1 \mu \mathrm{m}$ and $\gamma=707$, one obtains $u_{\mathrm{T}}=25 \mu \mathrm{m} \mathrm{s}^{-1}$ and the particle response time is found to be $T_{\mathrm{p}}=2.5 \mu \mathrm{s}$. For the driver frequency of $1400 \mathrm{~Hz}$ (maximum frequency used in this study), the particle response time is more than 200 times shorter than the excitation period. Thus, it can be concluded that the flow of tracer particles accurately follows the acoustic wave motion.
The velocity vector fields were calculated in LaVision Davis 8.11 software which uses cross-correlation algorithms with interrogation window size of $32 \times 32$ pixels and a $50 \%$ overlap between adjacent interrogation areas.

\section{Results of investigation}

A series of PIV vector maps can show in details the evolution of the acoustic velocity field throughout the acoustic cycle in the near field region. As an example, six phases where chosen to present the evolution of the acoustic velocity at the tube end for monotonic excitation. Fig. 5 shows instantaneous, phase-locked images of acoustic field excited at $700 \mathrm{~Hz}(\lambda=0.49 \mathrm{~m}, T=14 \mathrm{~ms})$ and $1400 \mathrm{~Hz}(\lambda=0.246 \mathrm{~m}, T=0.7 \mathrm{~ms})$ realized for 6 phase steps equally distributed within one acoustic period.

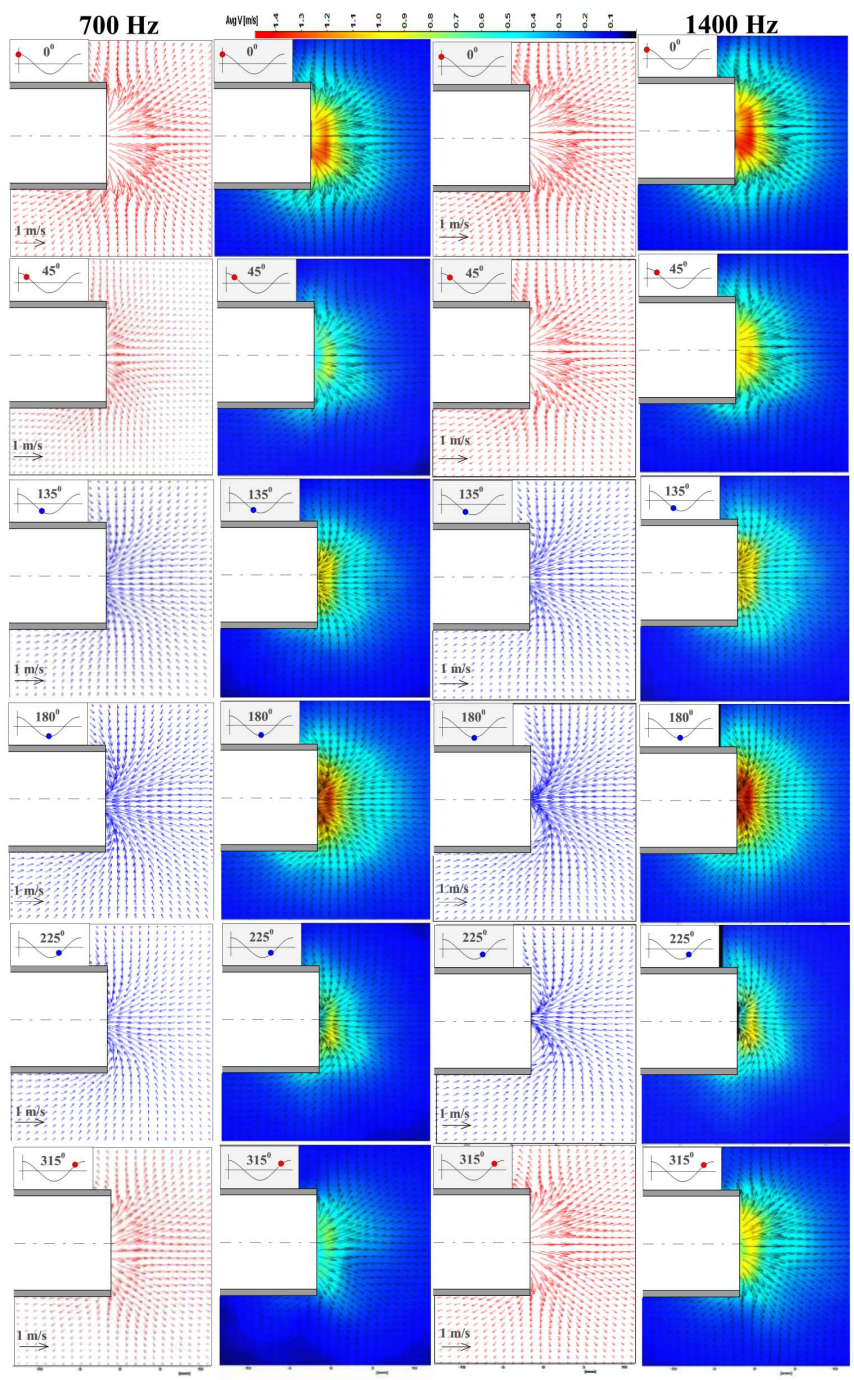

Fig. 5. Instantaneous acoustic velocity fields measured at six chosen phases of one acoustic cycle for $700 \mathrm{~Hz}$ tone (left two columns) and $1400 \mathrm{~Hz}$ tone (right columns).

It can be noticed that there is no symmetry in blowing (red) and suction (blue) phases, what also influences the shape of the radiated wave. These phenomena, well 
known from fluid mechanics, would be difficult to observe for acoustic excitation without new laser measurement techniques. Using them can provide new insights in properties of acoustical sources.

The development of interaction between directional acoustic flow on its way between the outlet of the tube and the exhausted sound field can be observed over the course of a single acoustic cycle. This provides a better understanding than that which is offered by acoustic streaming measurements alone or by other flow visualization techniques. In the case of the open ended tube, as a conversely directed acoustic wave reaches the open end it encounters an abrupt change in acoustical conditions resulting in a sudden change in acoustic impedance. A part of the sound wave is reflected back into the tube and a part is being transmitted forward as freely propagating sound (or noise). This phenomenon is attributed to changes in the acoustic impedance at the opening of the tube often called the radiation impedance or termination impedance [18]. Profile of the open end of tube has a significant effect on the changes of the terminating impedance. A qualitative evaluation of the physical flow mechanism contributing to the observed losses can be made by comparison of the termination impedance results with PIV vector maps.

\section{Concluding remarks}

This publication shows an application of optical PIV method to measure and visualize the instantaneous acoustic velocity field over an area around termination of a circular tube. Tests have confirmed that the monotonic sound flow with an acoustic velocity distribution can be measured and graphically illustrated using the non-invasive measurement technique even if the image recording rate of the system is insufficient in terms of sampling rate. Phase-locked particle image velocimetry (PIV) is a method which allows to perform non-intrusive instantaneous measurement of acoustic velocity field and acoustic wave propagation by synchronizing the image pairs acquisition with excitation signal. Until recently the PIV technique was extensively used only in mechanical fluid dynamics [19, 20] and now, with proper adaptation without main changes of lasers and camera equipment, it may be used to measure phenomena generated by motion of acoustic particles inside an acoustic flow field. The phase-locked acoustic PIV (A-PIV) method can be used to build an acoustic particle velocity map for each chosen phase of the exciting signal with the minimum disturbance of the sound field. As such, it has many applications in situations in which there is either a sufficiently high-intensity sound field only or a superposition of acoustic and mass flow. One of such situations is the evaluation of real boundary conditions for numerical simulations, where the lack of conformity with measurement is frequently caused by wrong assumptions concerning initial and boundary conditions. As it has been shown above, the open end of a pipe in the near field is not a perfect monopole or dipole, so simulations with ideal sound sources models can not exactly reflect the real behavior of acoustic systems with this kind of sources. The fusion of the actual measurement data of particle velocity distribution in time and space domain around the acoustic source and the numerical model of sound propagation can provide better solutions.

\section{References}

[1] J. Sung, J.Y. Yoo, Meas. Sci. Technol. 12, 655 (2001).

[2] D.B. Hann, C.A. Greated, Meas. Sci. Technol. 8, 1517 (1997).

[3] S.C. Roh, S.O. Park, Experiments in Fluids 34, 63 (2003).

[4] A. Henning, K. Kaepernick, K. Ehrenfried, L. Koop, A. Dillmann, Experiments in Fluids 45, 1073 (2008).

[5] R. Macdonald, D. Skulina, M. Campbell, J-Ch. Valerie, D. Marx, H. Bailliet, 10-eme Congres Francais d'Acoustique, Lyon 2010.

[6] J. Westerweel, Meas. Sci. Technol. 8, 1379 (1997).

[7] M.J. Lighthill, Proc. R. Soc. A 211, 564 (1952).

[8] M.S. Howe, Theory of Vortex Sound, Cambridge Univ. Press, Cambridge 2003.

[9] S. Weyna, W. Mickiewicz, M. Pyła, M. Jabłoński, Arch. Acous. 38(2), 217 (2013).

[10] F.D. Marx, H. Bailliet, J.-Ch. Valiere, Acta Acust. with Acust. 94, 54 (2008).

[11] F.J. Fahy, Sound Intensity, CRC Press, London 1995.

[12] S. Weyna, XX Fluid Mechanics Conf., S27-2, 2012.

[13] R.J. Adrian, Exp. Fluids 39, 159 (2005).

[14] M. Raffel, C. Willert, J. Kompenhans, Particle image velocimetry: a practical guide, Springer, New York 2002.

[15] S. Moreau, H. Bailliet, J-Ch. Valiere, R. Boucheron, G. Poignand, Acta Acust. with Acust. 95, 805 (2009).

[16] W.H. Snyder, J.L. Lumley, J. Fluid Mech. 48, 41 (1971).

[17] D.A. Siegel, A.J. Plueddemann, J. Atmos. Ocean. Technol. 8, 296 (1991).

[18] J.P. Dalmont, C.J. Nederveen, N. Joly, J. Sound Vib. 244(3), 505 (2001).

[19] B.W. Oudheusden, F. Scarano, Exp. Fluids 39, 86 (2005).

[20] L. Laurenco, S. Subramanian, Z. Ding, Meas. Sci. Technol. 8, 1533 (1997). 